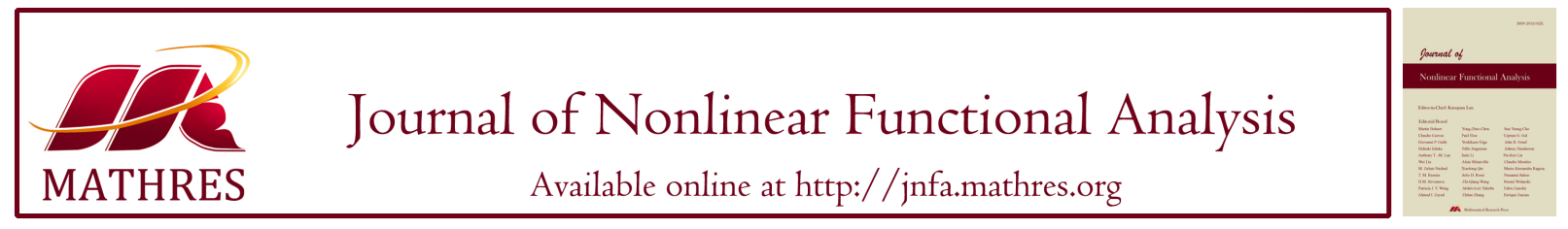

\title{
METRIC SPACES WITH ASYMPTOTIC PROPERTY $C$ AND FINITE DECOMPOSITION COMPLEXITY
}

\author{
JINGMING ZHU, YAN WU* \\ College of Data Science, Jiaxing University, Jiaxing 314001, China
}

\begin{abstract}
We construct a class of metric spaces $X_{\omega+k}$ whose transfinite asymptotic dimension and complementary-finite asymptotic dimension are both $\omega+k$ for any $k \in \mathbb{N}$, where $\omega$ is the smallest infinite ordinal number and a metric space $Y_{2 \omega}$ whose transfinite asymptotic dimension and complementary-finite asymptotic dimension are both $2 \omega$. Finally, we introduce a geometric property called decomposition dimension (decodim). Using decomposition dimension, we prove that the metric spaces $X_{\omega+k}$ and $Y_{2 \omega}$ have finite decomposition complexity.

Keywords. Asymptotic property $C$; Complementary-finite asymptotic dimension; Finite decomposition complexity; Transfinite asymptotic dimension;.
\end{abstract}

\section{INTRODUCTION}

The asymptotic dimension is a coarse invariant of metric spaces introduced by Gromov [1]. Recently, it has received a great deal of attention; see, e.g., $[2,3,4,5]$ and the references therein. Generalizing finite asymptotic dimension, Dranishnikov introduced the notion of the asymptotic property $C$ in [6], which covers a large family of metric spaces with the infinite asymptotic dimension. In 2010, Radul generalized the asymptotic dimension of a metric space $X$ to the transfinite asymptotic dimension, which is denoted by $\operatorname{trasdim}(X)$, and proved that, for a metric space $X, X$ has the asymptotic property $C$ is equivalent to $\operatorname{trasdim}(X)<\infty$ [7]. There are examples of the metric space with trasdim $=\infty$ and the metric space with trasdim $=\omega$, where $\omega$ is the smallest infinite ordinal number [7]. But, if there is a metric space $X$ with $\omega<\operatorname{trasdim}(X)<\infty$ was unknown until we constructed a metric space whose transfinite asymptotic dimension is $\omega+1$ [8]. In [9], we introduced another approach to classify the metric spaces with the infinite asymptotic dimension, which is called the complementary-finite asymptotic dimension (coasdim).

${ }^{*}$ Corresponding author.

E-mail addresses: jingmingzhu@ mail.zjxu.edu.cn (J. Zhu), yanwu@ mail.zjxu.edu.cn (Y. Wu).

Received April 13, 2021; Accepted May 7, 2021.

(C)2021 Journal of Nonlinear Functional Analysis 
Inspired by the method in [8], we construct a class of metric spaces $X_{\omega+k}$ with

$$
\operatorname{trasdim}\left(X_{\omega+k}\right)=\operatorname{coasdim}\left(X_{\omega+k}\right)=\omega+k \text { for any } k \in \mathbb{N},
$$

which generalizes the result in [8]. We use these metric spaces to construct a metric space $Y_{2} \omega$ with $\operatorname{trasdim}\left(Y_{2 \omega}\right)=$ coasdim $\left(Y_{2 \omega}\right)=2 \omega$.

In 2012, Guentner, Tessera and $\mathrm{Yu}$ [10] introduced the notion of finite decomposition complexity to study the topological rigidity of manifolds, and they proved that every metric space of bounded geometry with finite decomposition complexity has property $A$ [11]. Dranishnikov proved that every metric space of bounded geometry with asymptotic property $\mathrm{C}$ has property $A$ [6]. The relation between asymptotic property $C$ and finite decomposition complexity was studied by Dranishnikov and Zarichnyi [5]. There is no example of group known which makes a difference between asymptotic property $C$ and the finite decomposition complexity. Here we prove that $X_{\omega+k} \in \mathscr{D}_{\omega+k}$, and $Y_{2 \omega} \in \mathscr{D}_{2 \omega}$. Therefore, $X_{\omega+k}$ and $Y_{2 \omega}$ have both asymptotic property $C$, and the finite decomposition complexity.

\section{PRELIMINARIES}

Let $(X, d)$ be a metric space, and $U, V \subseteq X$. Define

$$
\operatorname{diam} U=\sup \{d(x, y) \mid x, y \in U\} \text { and } d(U, V)=\inf \{d(x, y) \mid x \in U, y \in V\} .
$$

Let $R$ and $r$ be positive numbers, and let $\mathscr{U}$ be a family of subsets of $X$. We say that $\mathscr{U}$ is $R$-bounded if

$$
\operatorname{diam} \mathscr{U} \doteq \sup \{\operatorname{diam} U \mid U \in \mathscr{U}\} \leq R .
$$

In this case, $\mathscr{U}$ is said to be uniformly bounded. We say that $\mathscr{U}$ is $r$-disjoint if

$$
d(U, V) \geq r \text { for every } U, V \in \mathscr{U} \text { with } U \neq V .
$$

Let $A$ be a subset of $X$ and $\varepsilon>0$. Define

$$
N_{\varepsilon}(A)=\{x \in X \mid d(x, A)<\varepsilon\} \text { and } \overline{N_{\varepsilon}(A)}=\{x \in X \mid d(x, A) \leq \varepsilon\} .
$$

Letting $\delta>0$, we denote

$$
N_{\delta}(\mathscr{U})=\left\{N_{\delta}(U) \mid U \in \mathscr{U}\right\}
$$

and

$$
\bigcup \mathscr{U}=\bigcup\{U \mid U \in \mathscr{U}\} \text { and } \mathscr{U}_{1} \cup \mathscr{U}_{2}=\left\{U \mid U \in \mathscr{U}_{1} \text { or } U \in \mathscr{U}_{2}\right\} .
$$

Definition 2.1. [4] The asymptotic dimension of a metric space $X$ does not exceed $n$ and write $\operatorname{asdim}(X) \leq n$ if there exists $n \in \mathbb{N}$ such that, for every $r>0$, there exists a sequence of uniformly bounded families $\left\{\mathscr{U}_{i}\right\}_{i=0}^{n}$ of subsets of $X$ such that $\bigcup_{i=0}^{n} \mathscr{U}_{i}$ covers $X$, and each $\mathscr{U}_{i}$ is $r$-disjoint for $i=0,1, \cdots, n$. In this case, we say that $X$ has finite asymptotic dimension.

We say that $\operatorname{asdim}(X)=n$ if $\operatorname{asdim}(X) \leq n$ and $\operatorname{asdim}(X) \leq n-1$ is not true.

We say that $\operatorname{asdim}(X)<\infty$ if $\operatorname{asdim}(X) \leq m$ for some $m \in \mathbb{N}$, and $\operatorname{asdim}(X)=\infty$ if $\operatorname{asdim}(X) \leq$ $n$ is not true for any $n \in \mathbb{N}$.

Definition 2.2. [7] Let $\operatorname{Fin} \mathbb{N}$ be the collection of all finite, nonempty subsets of $\mathbb{N}$, and let $M \subseteq F \operatorname{in} \mathbb{N}$. For $\sigma \in\{\varnothing\} \bigcup F i n \mathbb{N}$, let

$$
M^{\sigma}=\{\tau \in F i n \mathbb{N} \mid \tau \cup \sigma \in M \text { and } \tau \cap \sigma=\varnothing\} .
$$


Let $M^{a}$ abbreviate $M^{\{a\}}$ for $a \in \mathbb{N}$. Define the ordinal number Ord $M$ inductively as follows:

$$
\begin{aligned}
\operatorname{Ord} M=0 & \Leftrightarrow M=\varnothing, \\
\operatorname{Ord} M \leq \alpha & \Leftrightarrow \forall a \in \mathbb{N}, \text { there exists } \beta<\alpha, \text { such that } \operatorname{Ord} M^{a} \leq \beta, \\
\operatorname{Ord} M=\alpha & \Leftrightarrow \operatorname{Ord} M \leq \alpha \text { and } \operatorname{Ord} M \leq \beta \text { is not true for any } \beta<\alpha, \\
\operatorname{Ord} M=\infty & \Leftrightarrow \operatorname{Ord} M \leq \alpha \text { is not true for any ordinal number } \alpha .
\end{aligned}
$$

Definition 2.3. [7] Given a metric space $X$, let

$$
\begin{aligned}
A(X)=\{\sigma \in F i n \mathbb{N} \mid & \text { there are no uniformly bounded families } \mathscr{U}_{i} \text { for } i \in \sigma \\
& \text { such that each } \left.\mathscr{U}_{i} \text { is } i \text {-disjoint and } \bigcup_{i \in \sigma} \mathscr{U}_{i} \operatorname{covers} X\right\} .
\end{aligned}
$$

The transfinite asymptotic dimension of $X$ is defined as $\operatorname{trasdim}(X)=\operatorname{Ord} A(X)$.

Remark 2.4. Note that $\operatorname{trasdim}(X) \leq n$ if and only if $\operatorname{asdim}(X) \leq n$.

Lemma 2.5. (see [9], Proposition 2.1) Let $X$ be a metric space, and let $l \in \mathbb{N} \cup\{0\}$. Then the following conditions are equivalent:

(1) $\operatorname{trasdim}(X) \leq \omega+l$;

(2) for every $k \in \mathbb{N}$, there exists $m=m(k) \in \mathbb{N}$ such that for every $n \in \mathbb{N}$, there are uniformly bounded families $\mathscr{U}_{-l}, \mathscr{U}_{-l+1}, \cdots, \mathscr{U}_{m}$ satisfying $\mathscr{U}_{i}$ is $k$-disjoint for $i \in\{-l, \cdots, 0\}, \mathscr{U}_{j}$ is $n$-disjoint for $j \in\{1,2, \cdots, m\}$ and $\bigcup_{i=-l}^{m} \mathscr{U}_{i}$ covers $X$.

Definition 2.6. [9] Every ordinal number $\gamma$ can be represented as $\gamma=\lambda(\gamma)+n(\gamma)$, where $\lambda(\gamma)$ is the limit ordinal or 0 and $n(\gamma) \in \mathbb{N} \cup\{0\}$. Let $X$ be a metric space, we define complementaryfinite asymptotic dimension coasdim $(X)$ inductively as follows:

- $\operatorname{coasdim}(X)=-1$ if and only if $X=\emptyset$,

- $\operatorname{coasdim}(X) \leq \gamma$ if and only if for every $r>0$ there exist $r$-disjoint and uniformly bounded families $\mathscr{U}_{0}, \ldots, \mathscr{U}_{n(\gamma)}$ of subsets of $X$ such that coasdim $\left(X \backslash \bigcup\left(\bigcup_{i=0}^{n(\gamma)} \mathscr{U}_{i}\right)\right) \leq$ $\alpha$ for some $\alpha<\lambda(\gamma)$,

- $\operatorname{coasdim}(X)=\gamma$ if and only if $\operatorname{coasdim}(X) \leq \gamma$ and for any $\beta<\gamma, \operatorname{coasdim}(X) \leq \beta$ is not true.

Remark 2.7. Note that coasdim $(X) \leq n$ if and only if $\operatorname{asdim}(X) \leq n$.

Lemma 2.8. (see [9], Theorem 3.3) Let $X$ be a metric space with $X_{1}, X_{2} \subseteq X$. Then coasdim $\left(X_{1} \cup\right.$ $\left.X_{2}\right) \leq \max \left\{\operatorname{coasdim}\left(X_{1}\right)\right.$, coasdim $\left.\left(X_{2}\right)\right\}$.

Definition 2.9. Let $X$ and $Y$ be metric spaces. $f: X \rightarrow Y$ is said to be coarse embedding if there are nondecreasing functions $p_{1}, p_{2}: \mathbb{R}^{+} \rightarrow \mathbb{R}^{+}$such that $\lim _{x \rightarrow+\infty} p_{1}(x)=+\infty$ and for every $x_{1}, x_{2} \in X$

$$
p_{1}\left(d\left(x_{1}, x_{2}\right)\right) \leq d\left(f\left(x_{1}\right), f\left(x_{2}\right)\right) \leq p_{2}\left(d\left(x_{1}, x_{2}\right)\right) .
$$

If, additionally, there exists $R>0$ such that $Y \subseteq N_{R}(f[X])$, then $f$ is called coarse equivalence and metric spaces $X$ and $Y$ are said to be coarse equivalent. Coarse equivalence is an equivalence relation.

Transfinite asymptotic dimension and complementary-finite asymptotic dimension are coarsely invariant properties of metric spaces. 
Lemma 2.10. (see $[9,12])$ Let $X$ and $Y$ be metric spaces, and let $\xi$ be an countable ordinal number. There is a coarse embedding $\phi: X \rightarrow Y$ from $X$ to $Y$. If coasdim $(Y) \leq \xi$ and $\operatorname{trasdim}(Y) \leq \xi$, then

$$
\operatorname{coasdim}(X) \leq \text { coasdim }(Y) \text { and trasdim }(X) \leq \text { trasdim }(Y) .
$$

Consequently, if $X$ and $Y$ are coarse equivalent, coasdim $(Y) \leq \xi$, and trasdim $(Y) \leq \xi$, then

$$
\text { trasdim } X=\text { trasdim } Y \text { and coasdim } X=\text { coasdim } Y \text {. }
$$

\section{MAin Results}

3.1. A metric space with transfinite asymptotic dimension $\omega+k$. In this section, we will construct a metric space $X_{\omega+k}$, and prove that $\operatorname{trasdim}\left(X_{\omega+k}\right)=\omega+k$ for every $k \in \mathbb{N}$, which are inspired by the method in [8].

Definition 3.1. ([13]) Let $X$ be a metric space, and let $A, B$ be a pair of disjoint subsets of $X$. We say that a subset $L \subset X$ is a partition of $X$ between $A$ and $B$ if there exist open sets $U, W \subset X$ satisfying the following conditions

$$
A \subset U, B \subset W \text { and } X=U \sqcup L \sqcup W .
$$

Definition 3.2. Let $X$ be a metric space, and let $A, B$ be a pair of disjoint subsets of $X$. For any $\varepsilon>0$, we say that a subset $L \subset X$ is an $\varepsilon$-partition of $X$ between $A$ and $B$ if there exist open sets $U, W \subset X$ satisfying the following conditions

$$
A \subset U, B \subset W, X=U \sqcup L \sqcup W, d(L, A)>\varepsilon \text { and } d(L, B)>\varepsilon .
$$

Clearly, an $\varepsilon$-partition $L$ of $X$ between $A$ and $B$ is a partition of $X$ between $A$ and $B$.

Lemma 3.3. ([14]) Let $L_{0} \doteq[0, B]^{n}$ for some $B>0$. Let $F_{i}^{+}$and $F_{i}^{-}$be the pairs of opposite faces of $L_{0}$, where $i=1,2, \cdots, n$, and let $0<\varepsilon<\frac{1}{6} B$. For $k=1,2, \cdots, n$, let $\mathscr{U}_{k}$ be an $\varepsilon$-disjoint and $\frac{1}{3} B$-bounded family of subsets of $[0, B]^{n}$. Then there exists a decreasing sequence of closed sets $[0, B]^{n}=L_{0} \supset L_{1} \supset L_{2} \cdots \supset L_{n}$ such that $L_{k+1}$ is an $\varepsilon$-partition of $L_{k}$ between $F_{k+1}^{+} \cap L_{k}$ and $F_{k+1}^{-} \cap L_{k}$, and $L_{k+1} \subseteq L_{k} \cap\left(\bigcup \mathscr{U}_{k+1}\right)^{c}$ for $k=0,1,2, \cdots, n-1$.

To prove the main results, we will use a version of the Lebesgue theorem.

Lemma 3.4. (see [13], Lemma 1.8.19) Let $F_{i}^{+}, F_{i}^{-}$, where $i \in\{1, \ldots, n\}$, be the pairs of opposite faces of $I^{n} \doteq[0,1]^{n}$. If $I^{n}=L_{0}^{\prime} \supset L_{1}^{\prime} \supset \ldots \supset L_{n}^{\prime}$ is a decreasing sequence of closed sets such that $L_{i}^{\prime}$ is a partition of $L_{i-1}^{\prime}$ between $L_{i-1}^{\prime} \cap F_{i}^{+}$and $L_{i-1}^{\prime} \cap F_{i}^{-}$for $i \in\{1,2, \ldots, n\}$, then $L_{n}^{\prime} \neq \emptyset$.

As it is similar with [8], we will use the asymptotic union to construct examples.

Definition 3.5. Let $\left\{Z_{i}\right\}_{i=1}^{\infty}$ be a sequence of subspaces of a metric space $\left(Z, d_{Z}\right)$. Let

$$
X=\bigsqcup_{i=1}^{\infty}\left(0, \cdots, 0, Z_{i}, 0, \cdots\right) .
$$

For every $x, y \in X$, there exist unique $l, k \in \mathbb{N}, x_{l} \in Z_{l}$ and $y_{k} \in Z_{k}$ such that $x=\left(0, \cdots, 0, x_{l}, 0, \cdots\right)$ and $y=\left(0, \cdots, 0, y_{k}, 0, \cdots\right)$. Assume that $l \leq k$, put $c=0$ if $l=k$, and $c=l+(l+1)+\ldots+(k-$ $1)$ if $l<k$. Define a metric on $X$ by

$$
d(x, y)=d_{Z}\left(x_{l}, y_{k}\right)+c .
$$


We say that $(X, d)$ is asymptotic union of $\left\{Z_{i}\right\}_{i=1}^{\infty}$, which is denoted by as $\bigsqcup_{i=1}^{\infty} Z_{i}$. And we denote $a s \bigsqcup_{i=n}^{\infty} Z_{i}$ as a subspace of $a s \bigsqcup_{i=1}^{\infty} Z_{i}$.

For every $k, i \in \mathbb{N}$, let

$$
X_{\omega+k}^{(i)}=\left\{\left(x_{1}, \ldots, x_{i}\right) \in \mathbb{R}^{i}||\left\{j \mid x_{j} \notin 2^{i} \mathbb{Z}\right\} \mid \leq k\right\} .
$$

Note that $X_{\omega+k}^{(i)} \subset \mathbb{R}^{i}$ for each $i \in \mathbb{N}$. Let $X_{\omega+k}=$ as $\bigsqcup_{i=1}^{\infty} X_{\omega+k}^{(i)}$, where $X_{\omega+k}^{(i)}$ is a subspace of the metric space $\left(\bigoplus \mathbb{R}, d_{\max }\right)$ for each $i \in \mathbb{N}$, and $d_{\max }$ is the maximum metric.

Proposition 3.6. For any $k \in \mathbb{N}$, trasdim $\left(X_{\omega+k}\right) \leq \omega+k-1$ is not true.

Proof. Suppose that trasdim $\left(X_{\omega+k}\right) \leq \omega+k-1$. By Lemma 2.5, for every $n \in \mathbb{N}$, there exists $m=m(n) \in \mathbb{N}$ such that there exist $B$-bounded families $\mathscr{U}_{-k+1}, \mathscr{U}_{-k+2}, \ldots, \mathscr{U}_{m-1}, \mathscr{U}_{m}$ satisfying $\mathscr{U}_{i}$ is $n$-disjoint for $i=-k+1, \ldots, 0, \mathscr{U}_{j}$ is $2^{m+k+2}$-disjoint for $j=1,2, \ldots, m$ and $\bigcup_{i=-k+1}^{m} \mathscr{U}_{i}$ covers $X_{\omega+k}$ and hence covers $[0,6 B]^{m+k} \cap X_{\omega+k}^{(m+k)}$. Without lose of generality, we can assume $B=B(n)>\max \left\{n, 2^{m+k+2}\right\}$.

We assume that $p=\frac{6 B}{2^{m+k}} \in \mathbb{N}$. Taking a bijection $\psi:\left\{1,2, \cdots, p^{m+k}\right\} \rightarrow\{0,1,2, \cdots, p-$ $1\}^{m+k}$, let

$$
Q(t)=\prod_{j=1}^{m+k}\left[2^{m+k} \psi(t)_{j}, 2^{m+k}\left(\psi(t)_{j}+1\right)\right], \text { in which } \psi(t)_{j} \text { is the } j \text { th coordinate of } \psi(t) .
$$

Let $\mathscr{Q}=\left\{Q(t) \mid t \in\left\{1,2, \cdots, p^{m+k}\right\}\right\}$. Then, $[0,6 B]^{m+k}=\bigcup_{Q \in \mathscr{Q}} Q$. Note that

$$
[0,6 B]^{m+k} \cap X_{\omega+k}^{(m+k)}=\bigcup_{Q \in \mathscr{Q}} \partial_{k} Q
$$

where $\partial_{k} Q$ is the $k$-dimensional skeleton of $Q$.

Let $L_{0}=[0,6 B]^{m+k}$. By Lemma 3.3, since $N_{2^{m+k}}\left(\mathscr{U}_{1}\right)$ is $2^{m+k}$-disjoint and $\left(2^{m+k+1}+B\right)$ bounded, there exists a $2^{m+k}$-partition $L_{1}$ of $[0,6 B]^{m+k}$ such that

$$
L_{1} \subset\left(\bigcup N_{2^{m+k}}\left(\mathscr{U}_{1}\right)\right)^{c} \cap[0,6 B]^{m+k},
$$

and $d\left(L_{1}, F_{1}^{+/-}\right)>2^{m+k}$. Since $L_{1}$ is a $2^{m+k}$-partition of $[0,6 B]^{m+k}$ between $F_{1}^{+}$and $F_{1}^{-}$, then $[0,6 B]^{m+k}=L_{1} \sqcup A_{1} \sqcup B_{1}$ such that $A_{1}, B_{1}$ are open in $[0,6 B]^{m+k}$, and $A_{1}, B_{1}$ contain two opposite facets $F_{1}^{-}, F_{1}^{+}$respectively.

Let $\mathscr{M}_{1}=\left\{Q \in \mathscr{Q} \mid Q \cap L_{1} \neq \emptyset\right\}$, and $M_{1}=\bigcup \mathscr{M}_{1}$. Since $L_{1}$ is a $2^{m+k}$-partition of $[0,6 B]^{m+k}$ between $F_{1}^{+}$and $F_{1}^{-}$, then $M_{1}$ is a partition of $[0,6 B]^{m+k}$ between $F_{1}^{+}$and $F_{1}^{-}$, i.e., $[0,6 B]^{m+k}=$ $M_{1} \sqcup A_{1}^{\prime} \sqcup B_{1}^{\prime}$ such that $A_{1}^{\prime}$ and $B_{1}^{\prime}$ are open in $[0,6 B]^{m+k}$, and $A_{1}^{\prime}$ and $B_{1}^{\prime}$ contain two opposite facets $F_{1}^{-}, F_{1}^{+}$, respectively. Let

$$
L_{1}^{\prime}=\partial_{m+k-1} M_{1}=\bigcup\left\{\partial_{m+k-1} Q \mid Q \in \mathscr{M}_{1}\right\} .
$$

Then, $[0,6 B]^{m+k} \backslash\left(L_{1}^{\prime} \sqcup A_{1}^{\prime} \sqcup B_{1}^{\prime}\right)$ is the union of some disjoint open $(m+k)$-dimensional cubes with length of edge $=2^{m+k}$. So $L_{1}^{\prime}$ is a partition of $[0,6 B]^{m+k}$ between $F_{1}^{+}$and $F_{1}^{-}$, and $L_{1}^{\prime} \subset$ $\left(\bigcup \mathscr{U}_{1}\right)^{c} \cap[0,6 B]^{m+k}$.

For $N_{2^{m+k}}\left(\mathscr{U}_{2}\right)$, there exists a $2^{m+k}$-partition $L_{2}$ of $L_{1}^{\prime}$ such that

$$
L_{2} \subset\left(\bigcup N_{2^{m+k}}\left(\mathscr{U}_{2}\right)\right)^{c} \cap[0,6 B]^{m+k},
$$


and $d\left(L_{2}, F_{2}^{+/-}\right)>2^{m+k}$. Since $L_{2}$ is a $2^{m+k}$-partition of $L_{1}^{\prime}$ between $L_{1}^{\prime} \cap F_{2}^{+}$and $L_{1}^{\prime} \cap F_{2}^{-}$, then $L_{1}^{\prime}=L_{2} \sqcup A_{2} \sqcup B_{2}$ such that $A_{2}$ and $B_{2}$ are open in $L_{1}^{\prime}$, and $A_{2}$ and $B_{2}$ contain two opposite facets $L_{1}^{\prime} \cap F_{2}^{-}, L_{1}^{\prime} \cap F_{2}^{+}$, respectively, and $d\left(L_{2}, F_{2}^{+/-}\right)>2^{m+k}$.

Let $\mathscr{M}_{2}=\left\{Q \in \mathscr{M}_{1} \mid Q \cap L_{2} \neq \emptyset\right\}$, and $M_{2}=\bigcup \mathscr{M}_{2}$. Since $L_{2}$ is a $2^{m+k}$-partition of $L_{1}^{\prime}$ between $L_{1}^{\prime} \cap F_{2}^{+}$and $L_{1}^{\prime} \cap F_{2}^{-}$, then $M_{2} \cap L_{1}^{\prime}$ is a partition of $L_{1}^{\prime}$ between $L_{1}^{\prime} \cap F_{2}^{+}$and $L_{1}^{\prime} \cap F_{2}^{-}$, i.e., $L_{1}^{\prime}=\left(M_{2} \cap L_{1}^{\prime}\right) \sqcup A_{2}^{\prime} \sqcup B_{2}^{\prime}$ such that $A_{2}^{\prime}, B_{2}^{\prime}$ are open in $L_{1}^{\prime}$, and $A_{2}^{\prime}$ and $B_{2}^{\prime}$ contain two opposite facets $L_{1}^{\prime} \cap F_{2}^{-}$and $L_{1}^{\prime} \cap F_{2}^{+}$, respectively. Let

$$
L_{2}^{\prime}=\partial_{m+k-2} M_{2} \doteq \bigcup\left\{\partial_{m+k-2} Q \mid Q \in \mathscr{M}_{2}\right\} .
$$

Then $L_{1}^{\prime} \backslash\left(L_{2}^{\prime} \sqcup A_{2}^{\prime} \sqcup B_{2}^{\prime}\right)$ is the union of some disjoint open $(m+k-1)$-dimensional cubes with length of edge $=2^{m+k}$. So $L_{2}^{\prime}$ is also a partition of $L_{1}^{\prime}$ between $L_{1}^{\prime} \cap F_{2}^{+}$and $L_{1}^{\prime} \cap F_{2}^{-}$, and $L_{2}^{\prime} \subset\left(\bigcup\left(\mathscr{U}_{1} \cup \mathscr{U}_{2}\right)\right)^{c} \cap[0,6 B]^{m+k}$. After $m$ steps above, we have $L_{m}^{\prime}$ to be a partition of $L_{m-1}^{\prime}$ and $L_{m}^{\prime} \subset\left(\cup\left(\mathscr{U}_{1} \cup \ldots \cup \mathscr{U}_{m}\right)\right)^{c} \cap[0,6 B]^{m+k}$. Note that $L_{m}^{\prime} \subset X_{\omega+k}^{(m+k)}$ and hence

$$
L_{m}^{\prime} \subset\left(\bigcup\left(\mathscr{U}_{-k+1} \cup \ldots \cup \mathscr{U}_{0}\right)\right) \cap[0,6 B]^{m+k} .
$$

For $j=1,2, \cdots, k$, there exists a partition $L_{m+j}^{\prime}$ of $L_{m+j-1}^{\prime}$ between $L_{m+j-1}^{\prime} \cap F_{m+j}^{+}$and $L_{m+j-1}^{\prime} \cap$ $F_{m+j}^{-}$such that $L_{m+j}^{\prime} \subseteq L_{m+j-1}^{\prime} \cap\left(\bigcup\left(\mathscr{U}_{-j+1} \cup \ldots \cup \mathscr{U}_{m}\right)\right)^{c}$ due to Lemma 3.3. It follows that

$$
L_{m+k}^{\prime} \subseteq L_{m+k-1}^{\prime} \cap\left(\bigcup\left(\mathscr{U}_{-k+1} \cup \ldots \cup \mathscr{U}_{0}\right)\right)^{c}=\emptyset,
$$

which is a contradiction with Lemma 3.4. So trasdim $\left(X_{\omega+k}\right) \leq \omega+k-1$ is not true.

Lemma 3.7. (see [15], Proposition 3.1) Let $X$ be a metric space if coasdim $(X) \leq \gamma$ for some ordinal number $\gamma$, then trasdim $(X) \leq \gamma$.

For every $n, i, k \in \mathbb{N}$, let

$$
X_{\omega+k}^{(i, n)}=\left\{\left(x_{1}, \ldots, x_{i}\right) \in \mathbb{R}^{i}||\left\{j \mid x_{j} \notin 2^{n} \mathbb{Z}\right\} \mid \leq k\right\} .
$$

Note that $X_{\omega+k}^{(i)}=X_{\omega+k}^{(i, i)}$.

Lemma 3.8. For every $r \in \mathbb{N}$ with $r \geq 4$, there exist $n=r \in \mathbb{N}$ and $r$-disjoint uniformly bounded families $\mathscr{U}_{0}, \mathscr{U}_{1}$ such that $\mathscr{U}_{0} \cup \mathscr{U}_{1}$ covers as $\bigsqcup_{i=n}^{\infty} X_{\omega+1}^{(i, n)}$.

Proof. For every $r \in \mathbb{N}$ and $r \geq 4$, choose $n=r \in \mathbb{N}$. For every $i \geq n$, let

$$
\mathscr{U}_{0}^{(i)}=\left\{\left(\prod_{t=1}^{i}\left(n_{t} 2^{n}-r, n_{t} 2^{n}+r\right)\right) \cap X_{\omega+1}^{(i, n)} \mid n_{t} \in \mathbb{Z}\right\},
$$

and

$$
\begin{aligned}
\mathscr{U}_{1}^{(i)}= & \left\{\left(\prod_{t=1}^{j-1}\left(n_{t} 2^{n}-r, n_{t} 2^{n}+r\right) \times\left[n_{j} 2^{n}+r,\left(n_{j}+1\right) 2^{n}-r\right] \times\right.\right. \\
& \left.\left.\prod_{t=j+1}^{i}\left(n_{t} 2^{n}-r, n_{t} 2^{n}+r\right)\right) \cap X_{\omega+1}^{(i, n)} \mid n_{t} \in \mathbb{Z}, 1 \leq j \leq i\right\} .
\end{aligned}
$$

It is easy to see that $\mathscr{U}_{0}^{(i)}$ and $\mathscr{U}_{1}^{(i)}$ are $r$-disjoint and $2^{n}$-bounded families. Now, for every $x=$ $\left(x_{1}, \ldots, x_{i}\right) \in X_{\omega+1}^{(i, n)} \backslash\left(\bigcup \mathscr{U}_{0}^{(i)}\right)$, there exists unique $j \in\{1,2, \cdots, i\}$ such that $x_{j} \in\left[n_{j} 2^{n}+r,\left(n_{j}+\right.\right.$ 1) $\left.2^{n}-r\right]$. It follows that $x \in \mathscr{U}_{1}^{(i)}$. Therefore, $\mathscr{U}_{0}^{(i)} \cup \mathscr{U}_{1}^{(i)}$ covers $X_{\omega+1}^{(i, n)}$. Let $\mathscr{U}_{0}=\bigcup_{i \geq n} \mathscr{U}_{0}^{(i)}$, 
and $\mathscr{U}_{1}=\bigcup_{i \geq n} \mathscr{U}_{1}^{(i)}$. Since $d\left(X_{\omega+1}^{(i, n)}, X_{\omega+1}^{(j, n)}\right) \geq n=r$ for every $i, j \geq n$ and $i \neq j$, then $\mathscr{U}_{0}, \mathscr{U}_{1}$ are $r$-disjoint and $2^{n}$-bounded families such that $\mathscr{U}_{0} \cup \mathscr{U}_{1}$ covers $a s \bigsqcup_{i=n}^{\infty} X_{\omega+1}^{(i, n)}$.

Remark 3.9. By Lemma 3.8, for every $r \in \mathbb{N}$ and $r>1$, there exist $3 r$-disjoint and uniformly bounded families $\mathscr{U}_{0}, \mathscr{U}_{1}$ such that $\mathscr{U}_{0} \cup \mathscr{U}_{1}$ covers $a s \bigsqcup_{i=3 r}^{\infty} X_{\omega+1}^{(i, 3 r)}$. Let $\mathscr{V}_{0}=\left\{N_{r}(U) \mid U \in\right.$ $\left.\mathscr{U}_{0}\right\}, \mathscr{V}_{1}=\left\{N_{r}(U) \mid U \in \mathscr{U}_{1}\right\}$. Then $\mathscr{V}_{0}, \mathscr{V}_{1}$ are $r$-disjoint uniformly bounded families, and $\mathscr{V}_{0} \cup$ $\mathscr{V}_{1}$ covers $a s \bigsqcup_{i=3 r}^{\infty} N_{r}\left(X_{\omega+1}^{(i, n)}\right)$, where $N_{r}\left(X_{\omega+1}^{(i, n)}\right)$ is $r$-neighborhood of $X_{\omega+1}^{(i, n)}$ in $\mathbb{R}^{i}$. By the similar argument, we obtain the following Lemma.

Lemma 3.10. For every $r \in \mathbb{N}$ and $r>1$, there exist $n=3^{k-1} r \in \mathbb{N}$ and $r$-disjoint uniformly bounded families $\mathscr{U}_{0}, \mathscr{U}_{1}, \ldots, \mathscr{U}_{k}$ such that $\mathscr{U}_{0} \cup \mathscr{U}_{1} \cup \ldots \cup \mathscr{U}_{k}$ covers as $\bigsqcup_{i=n}^{\infty} X_{\omega+k}^{(i, n)}$.

Proof. We will prove it by induction on $k$. By Lemma 3.8, the result is true for $k=1$. Assume that the result is true for $k=m$. Then, for every $r \in \mathbb{N}$ and $r>1$, there exist $n=3^{m} r \in \mathbb{N}$ and $3 r$-disjoint uniformly bounded families $\mathscr{V}_{0}, \mathscr{V}_{1}, \ldots, \mathscr{V}_{m}$ such that $\mathscr{V}_{0} \cup \mathscr{V}_{1} \cup \ldots \cup \mathscr{V}_{m}$ covers $a s \bigsqcup_{i=n}^{\infty} X_{\omega+m}^{(i, n)}$. Now, for $k=m+1$, let

$$
\mathscr{U}_{0}=\left\{N_{r}(V) \mid V \in \mathscr{V}_{0}\right\}, \cdots, \mathscr{U}_{m}=\left\{N_{r}(V) \mid V \in \mathscr{V}_{m}\right\} .
$$

Then $\mathscr{U}_{0}, \mathscr{U}_{1}, \cdots, \mathscr{U}_{m}$ are $r$-disjoint and uniformly bounded families such that $\mathscr{U}_{0} \cup \mathscr{U}_{1} \cup \ldots \cup$ $\mathscr{U}_{m}$ covers $a s \bigsqcup_{i=n}^{\infty} N_{r}\left(X_{\omega+m}^{(i, n)}\right)$. Let

$$
\begin{aligned}
\mathscr{U}_{m+1}^{(i)}= & \left\{\left\{x_{t}\right\}_{t=1}^{j_{1}-1} \times\left[n_{j_{1}} 2^{n}+r,\left(n_{j_{1}}+1\right) 2^{n}-r\right] \times\left(x_{t}\right)_{t=j_{1}+1}^{j_{2}-1} \times\left[n_{j_{2}} 2^{n}+r,\left(n_{j_{2}}+1\right) 2^{n}-r\right]\right. \\
& \times\left\{x_{t}\right\}_{t=j_{2}+1}^{j_{3}-1} \times \cdots \times\left\{x_{t}\right\}_{t=j_{m}+1}^{j_{m+1}-1} \times\left[n_{j_{m+1}} 2^{n}+r,\left(n_{j_{m+1}}+1\right) 2^{n}-r\right] \times\left\{x_{t}\right\}_{t=j_{m+1}+1}^{i} \mid x_{t} \\
\in & \left.2^{n} \mathbb{Z}, n_{j_{k}} \in \mathbb{Z}, 1 \leq k \leq m+1\right\} .
\end{aligned}
$$

It is easy to see that $\mathscr{U}_{m+1}^{(i)}$ is $r$-disjoint and $2^{n}$-bounded. Note that, for every $i \geq n$,

$$
X_{\omega+m+1}^{(i, n)} \backslash \bigcup \mathscr{U}_{m+1}^{(i)} \subset N_{r}\left(X_{\omega+m}^{(i, n)}\right)
$$

Indeed, for any $x=\left\{x_{t}\right\}_{t=1}^{i} \in X_{\omega+m+1}^{(i, n)} \backslash \cup \mathscr{U}_{m+1}^{(i)},\left\{x_{t}\right\}_{t=1}^{i} \in X_{\omega+m+1}^{(i, n)}$ implies that there exists at most $m+1$ coordinates $x_{t}$ such that $x_{t} \notin 2^{n} \mathbb{Z}$ and $x \notin \cup \mathscr{U}_{m+1}^{(i)}$ implies that, among all the $x_{t}$ with $x_{t} \notin 2^{n} \mathbb{Z}$, there exists at least one $x_{t_{0}}$ such that $d\left(x_{t_{0}}, 2^{n} \mathbb{Z}\right)<r$. It follows that $x \in N_{r}\left(X_{\omega+m}^{(i, n)}\right)$. Since

$$
d\left(X_{\omega+m+1}^{(i, n)}, X_{\omega+m+1}^{(j, n)}\right)>r \text { for every } i, j \geq n \text { and } i \neq j,
$$

then $\mathscr{U}_{m+1} \doteq \bigcup_{i \geq n} \mathscr{U}_{m+1}^{(i)}$ is an $r$-disjoint uniformly bounded family of subsets and

$$
a s \bigsqcup_{i=n}^{\infty} X_{\omega+m+1}^{(i, n)} \subset\left(\bigcup \mathscr{U}_{m+1}\right) \cup \bigcup_{i=n}^{\infty} N_{r}\left(X_{\omega+m}^{(i, n)}\right) \text {. }
$$

Therefore, $\mathscr{U}_{0} \cup \mathscr{U}_{1} \cup \ldots \cup \mathscr{U}_{m+1}$ covers $a s \bigsqcup_{i=n}^{\infty} X_{\omega+m+1}^{(i, n)}$. So the result is true for $k=m+1$.

Proposition 3.11. coasdim $\left(X_{\omega+k}\right) \leq \omega+k$.

Proof. For every $r>0$, by Lemma 3.10, there exist $n=n(r) \in \mathbb{N}$ and $r$-disjoint uniformly bounded families $\mathscr{U}_{0}, \mathscr{U}_{1}, \ldots, \mathscr{U}_{k}$ such that $\mathscr{U}_{0} \cup \mathscr{U}_{1} \cup \ldots \cup \mathscr{U}_{k}$ covers $a s \bigsqcup_{i=n}^{\infty} X_{\omega+k}^{(i, n)}$. Since 
$X_{\omega+k}^{(i)}=X_{\omega+k}^{(i, i)} \subset X_{\omega+k}^{(i, n)}$ for $i \geq n, X_{\omega+k} \backslash \bigcup\left(\mathscr{U}_{0} \cup \ldots \mathscr{U}_{k}\right) \subseteq a s \bigsqcup_{i=1}^{n-1} X_{\omega+k}^{(i)}$. From Lemma 2.8, one has

$$
\begin{array}{r}
\operatorname{coasdim}\left(X_{\omega+k} \backslash \bigcup\left(\mathscr{U}_{0} \cup \ldots \mathscr{U}_{k}\right)\right) \leq \operatorname{coasdim}\left(\text { as } \bigsqcup_{i=1}^{n-1} X_{\omega+k}^{(i)}\right) \leq \\
\text { coasdim }\left(\text { as } \bigsqcup_{i=1}^{n-1} \mathbb{R}^{i}\right) \leq \operatorname{coasdim}\left(\mathbb{R}^{n-1}\right)<\omega .
\end{array}
$$

It follows from the definition that $\operatorname{coasdim}\left(X_{\omega+k}\right) \leq \omega+k$.

Theorem 3.12. trasdim $\left(X_{\omega+k}\right)=\omega+k$ for every $k \in \mathbb{N}$.

Proof. From Lemma 3.7 and Proposition 3.11, one has $\operatorname{trasdim}\left(X_{\omega+k}\right) \leq \omega+k$. Using Proposition 3.6, we obtain that $\operatorname{trasdim}\left(X_{\omega+k}\right)=\omega+k$.

Theorem 3.13. coasdim $\left(X_{\omega+k}\right)=\omega+k$ for every $k \in \mathbb{N}$.

Proof. From Proposition 3.6 and Lemma 3.7, we can obtain that coasdim $\left(X_{\omega+k}\right) \leq \omega+k-1$ is not true. Then coasdim $\left(X_{\omega+k}\right)=\omega+k$ due to Proposition 3.11.

3.2. A metric space with complementary-finite asymptotic dimension and transfinite asymptotic dimension $2 \omega$. In this section, we will construct a metric space $Y_{2 \omega}$ by taking asymptotic union of all the metric spaces $Y_{\omega+k}$, which is coarsely equivalent to $X_{\omega+k}$ for any $k \in \mathbb{N}$.

For every $k, i \in \mathbb{N}$, let

$$
Y_{\omega+k}^{(i)}=\left\{\left(x_{1}, \ldots, x_{i}\right) \in\left(2^{k} \mathbb{Z}\right)^{i}||\left\{j \mid x_{j} \notin 2^{i} \mathbb{Z}\right\} \mid \leq k\right\}, Y_{\omega+k}=\operatorname{as} \bigsqcup_{i=1}^{\infty} Y_{\omega+k}^{(i)} \text { and } Y_{2 \omega}=\text { as } \bigsqcup_{k=1}^{\infty} Y_{\omega+k},
$$

where $Y_{\omega+k}$ is a subspace of the metric space as $\bigsqcup_{j=1}^{\infty} \mathbb{R}^{j}$ for each $j \in \mathbb{N}$.

Proposition 3.14. coasdim $\left(Y_{2 \omega}\right)=2 \omega$ and trasdim $\left(Y_{2 \omega}\right)=2 \omega$.

Proof. For any $k \in \mathbb{N}$, since $Y_{\omega+k} \subseteq X_{\omega+k}$ and $X_{\omega+k} \subseteq N_{2^{k}}\left(Y_{\omega+k}\right), Y_{\omega+k}$ and $X_{\omega+k}$ are coarse equivalent. It follows that

$$
\operatorname{trasdim}\left(Y_{\omega+k}\right)=\operatorname{trasdim}\left(X_{\omega+k}\right)=\omega+k \text { and coasdim }\left(Y_{\omega+k}\right)=\operatorname{coasdim}\left(X_{\omega+k}\right)=\omega+k
$$

due to Lemma 2.10. It follows that coasdim $\left(Y_{2 \omega}\right) \geq 2 \omega$ and $\operatorname{trasdim}\left(Y_{2 \omega}\right) \geq 2 \omega$.

For every $n>0$, let

$$
\mathscr{U}=\left\{\{x\} \mid x \in \text { as } \bigsqcup_{k=n+1}^{\infty} Y_{\omega+k}\right\} .
$$

Then $\mathscr{U}$ is $n$-disjoint and uniformly bounded, and

$$
Y_{2 \omega} \backslash \bigcup \mathscr{U}=a s \bigsqcup_{k=1}^{n} Y_{\omega+k}
$$

It follows from Lemma 2.8 that

$$
\operatorname{coasdim}\left(\text { as } \bigsqcup_{k=1}^{n} Y_{\omega+k}\right) \leq \operatorname{coasdim}\left(\text { as } \bigsqcup_{k=1}^{n} X_{\omega+k}\right) \leq \max _{1 \leq k \leq n}\left\{\operatorname{coasdim}\left(X_{\omega+k}\right)\right\}=\omega+n<2 \omega
$$


thus coasdim $\left(Y_{2 \omega} \backslash \cup \mathscr{U}\right)<2 \omega$. Then by the definition of complementary-finite asymptotic dimension, coasdim $\left(Y_{2 \omega}\right) \leq 2 \omega$. Hence $\operatorname{trasdim}\left(Y_{2 \omega}\right) \leq 2 \omega$, which is due to Lemma 3.7. Therefore, $\operatorname{coasdim}\left(Y_{2 \omega}\right)=2 \omega$, and $\operatorname{trasdim}\left(Y_{2 \omega}\right)=2 \omega$.

\subsection{The decomposition dimension of metric spaces.}

Definition 3.15. ([4]) We say that the metric family $\left\{X_{\alpha}\right\}$ satisfies the inequality $\operatorname{asdim}\left(X_{\alpha}\right) \leq n$ uniformly if, for every $r>0$, there exists $R>0$ such that, for each $\alpha$, there are $r$-disjoint and $R$-bounded families $\mathscr{U}_{0}^{\alpha}, \mathscr{U}_{1}^{\alpha}, \cdots, \mathscr{U}_{n}^{\alpha}$ of subsets of $X_{\alpha}$ such that $\bigcup_{i=0}^{n} \mathscr{U}_{i}^{\alpha}$ covers $X_{\alpha}$.

Now we introduce a new dimension in coarse geometry.

Definition 3.16. Every ordinal number $\gamma$ can be represented as $\gamma=\lambda(\gamma)+n(\gamma)$, where $\lambda(\gamma)$ is the limit ordinal or 0 , and $n(\gamma) \in \mathbb{N} \cup\{0\}$. For a metric space $X$, we define decomposition dimension $\operatorname{decodim}(X)$ inductively as follows:

- for $\gamma=n \in \mathbb{N}, \operatorname{decodim}(X) \leq n \Leftrightarrow \operatorname{coadim}(X) \leq n$;

- for $\gamma=\omega, \operatorname{decodim}(X) \leq \omega \Leftrightarrow \operatorname{coadim}(X) \leq \omega$;

- for $\gamma>\omega$, decodim $(X) \leq \gamma \Leftrightarrow$ for every $r>0$, there is an $r$-disjoint family $\mathscr{U}=$ $\left\{U_{i}\right\}$ of subsets of $X$ such that $\operatorname{asdim}\left(U_{i}\right) \leq n(\gamma)$ uniformly and $\operatorname{decodim}(X \backslash \bigcup \mathscr{U}) \leq$ $\alpha$ for some $\alpha<\lambda(\gamma)$;

- $\operatorname{decodim}(X)=\gamma \Leftrightarrow \operatorname{decodim}(X) \leq \gamma$ and $\operatorname{decodim}(X) \leq \beta$ is not true for any ordinal number $\beta<\gamma$.

Remark 3.17. It is easy to see that coasdim $(X) \leq \omega$ implies $\operatorname{decodim}(X)=\operatorname{coasdim}(X)$.

Lemma 3.18. Let $X$ and $Y$ be metric spaces, and let $\left\{Y_{\alpha}\right\}$ be metric family of subsets of $Y$ and $\operatorname{asdim}\left(Y_{\alpha}\right) \leq n$ uniformly. Let $\phi: X \rightarrow Y$ be a coarse embedding from $X$ to $Y$. Then, for the metric family $\left\{\phi^{-1}\left(Y_{\alpha}\right)\right\}$, asdim $\left(\phi^{-1}\left(Y_{\alpha}\right)\right) \leq n$ uniformly.

Proof. Since $\phi: X \rightarrow Y$ is a coarse embedding, there are nondecreasing functions $p_{1}, p_{2}: \mathbb{R}^{+} \rightarrow$ $\mathbb{R}^{+}$such that $\lim _{x \rightarrow+\infty} p_{1}(x)=+\infty$ and for every $x_{1}, x_{2} \in X$,

$$
p_{1}\left(d\left(x_{1}, x_{2}\right)\right) \leq d\left(\phi\left(x_{1}\right), \phi\left(x_{2}\right)\right) \leq p_{2}\left(d\left(x_{1}, x_{2}\right)\right) .
$$

For every $r>0$, asdim $\left(Y_{\alpha}\right) \leq n$ uniformly implies there exists $R>0$ such that, for each $\alpha$, there are $\left(p_{2}(r)+1\right)$-disjoint and $R$-bounded families $\mathscr{U}_{0}^{\alpha}, \mathscr{U}_{1}^{\alpha}, \cdots, \mathscr{U}_{n}^{\alpha}$ of subsets of $Y_{\alpha}$ with $\bigcup_{i=0}^{n} \mathscr{U}_{i}^{\alpha}$ covers $Y_{\alpha}$. Since $\lim _{x \rightarrow+\infty} p_{1}(x)=+\infty$, there exists $S>0$ such that $p_{1}(S)>R$. For $i=0,1,2, \cdots, n$, let

$$
\mathscr{V}_{i}^{\alpha}=\left\{\phi^{-1}(U) \mid U \in \mathscr{U}_{i}^{\alpha}\right\} .
$$

Then $\mathscr{V}_{i}^{\alpha}$ is $r$-disjoint and $S$-bounded families of subsets of $\phi^{-1}\left(Y_{\alpha}\right)$ with $\bigcup_{i=0}^{n} \mathscr{V}_{i}^{\alpha}$ covers $\phi^{-1}\left(Y_{\alpha}\right)$. So asdim $\left(\phi^{-1}\left(Y_{\alpha}\right)\right) \leq n$ uniformly.

Proposition 3.19. Let $X$ and $Y$ be metric spaces with decodim $(Y) \leq \xi$ for some countable ordinal number $\xi$. If there is a coarse embedding $\phi: X \rightarrow Y$ from $X$ to $Y$, then decodim $(X) \leq$ $\operatorname{decodim}(Y)$. Consequently, if $X$ and $Y$ are coarsely equivalent, then decodim $(X)=\operatorname{decodim}(Y)$.

Proof. We will prove it by induction on $\xi$. 
- For $\xi \leq \omega$, $\operatorname{decodim}(Y) \leq \xi \leq \omega$ implies coasdim $(Y) \leq \omega$. By Lemma 2.10,

$$
\operatorname{coasdim}(X) \leq \operatorname{coasdim}(Y) \leq \omega .
$$

It follows that

$$
\operatorname{decodim}(X)=\operatorname{coasdim}(X) \leq \operatorname{coasdim}(Y)=\operatorname{decodim}(Y) .
$$

- Assume that the statement is true for every $\xi<\beta$. Now let us consider the case of $\xi=\beta$ with $\beta>\omega$. Since $\phi: X \rightarrow Y$ is a coarse embedding, there are nondecreasing functions $p_{1}, p_{2}: \mathbb{R}^{+} \rightarrow \mathbb{R}^{+}$such that $\lim _{x \rightarrow+\infty} p_{1}(x)=+\infty$ and for every $x_{1}, x_{2} \in X$,

$$
p_{1}\left(d\left(x_{1}, x_{2}\right)\right) \leq d\left(\phi\left(x_{1}\right), \phi\left(x_{2}\right)\right) \leq p_{2}\left(d\left(x_{1}, x_{2}\right)\right) \text {. }
$$

For every $r>0$, there is a $\left(p_{2}(r)+1\right)$-disjoint family $\mathscr{U}=\left\{U_{\alpha}\right\}$ of subsets of $Y$ such that

$\operatorname{asdim}\left(U_{\alpha}\right) \leq n(\beta)$ uniformly and $\operatorname{decodim}(Y \backslash \bigcup \mathscr{U}) \leq \eta$ for some $\eta<\lambda(\beta)$.

Then $\operatorname{asdim}\left(\phi^{-1}\left(U_{\alpha}\right)\right) \leq n(\beta)$ uniformly by Lemma 3.18. Let $\mathscr{V}=\left\{\phi^{-1}\left(U_{\alpha}\right)\right\}$. Since the restriction of $\phi$ to $X \backslash \bigcup \mathscr{V}$ is a coarse embedding into $Y \backslash \cup \mathscr{U}$ and $\operatorname{decodim}(Y \backslash$ $\bigcup \mathscr{U}) \leq \eta, \operatorname{decodim}(X \backslash \bigcup \mathscr{V}) \leq \eta$ by induction hypothesis. So $\operatorname{decodim}(X) \leq \beta=$ $\operatorname{decodim}(Y)$.

Example 3.20. For $n \in \mathbb{N}$, $\operatorname{decodim}\left(X_{\omega+n}\right) \leq \omega+n$.

Proof. $\quad$ For $n=1$, by Theorem 3.13, coasdim $\left(X_{\omega+1}\right)=\omega+1$. Then coasdim $\left(X_{\omega+1}\right) \leq \omega$ is not true and hence $\operatorname{decodim}\left(X_{\omega+1}\right) \leq \omega$ is not true. Now it suffices to show that $\operatorname{decodim}\left(X_{\omega+1}\right) \leq \omega+1$. For any $k>0$ and integer $n>2 k$, let $\mathscr{V}_{0}^{(n)}=\left\{\left[i 2^{n}+k,(i+\right.\right.$ 1) $\left.\left.2^{n}-k\right] \mid i \in \mathbb{Z}\right\}$, and let

$$
\mathscr{W}_{0}^{(n)}=\left\{\prod_{i=1}^{j-1}\left\{n n_{i}\right\} \times V_{j} \times \prod_{i=j+1}^{n}\left\{n n_{i}\right\} \mid n_{i} \in \mathbb{Z}, V_{j} \in \mathscr{V}_{0}^{(n)}, j=1,2,3, \cdots, n\right\}
$$

Let $\mathscr{W}_{0}=\bigcup_{n>k} \mathscr{W}_{0}^{(n)}$. Then $\mathscr{W}_{0}$ is $k$-disjoint, and $\operatorname{asdim}(W) \leq 1$ uniformly for any $W \in \mathscr{W}_{0}$. It is easy to see $X_{\omega+1} \backslash \bigcup \mathscr{W}_{0} \subseteq N_{k}\left(X_{\omega}\right)$. Hence $\operatorname{coasdim}\left(X_{\omega+1} \backslash \bigcup \mathscr{W}_{0}\right) \leq \omega$, which implies $\operatorname{decodim}\left(X_{\omega+1} \backslash \bigcup \mathscr{W}_{0}\right) \leq \omega$. Therefore $\operatorname{decodim}\left(X_{\omega+1}\right) \leq \omega+1$.

- Assuming $\operatorname{decodim}\left(X_{\omega+k}\right) \leq \omega+k$ holds for $k \leq n-1$, we have that, for any $r>n>0$, the $r$-neighborhood $N_{r}\left(X_{\omega+n-1}\right)$ of $X_{\omega+n-1}$ in $X_{\omega+n}$ has $\operatorname{decodim}\left(N_{r}\left(X_{\omega+n-1}\right)\right) \leq \omega+$ $n-1$ due to Proposition 3.19. Let $i_{0} \in \mathbb{N}$ be the smallest number with $r \leq i_{0}$. For any $i \geq i_{0}$, for any subset $F \subset\{1, \cdots, i\}$ with $|F|=n$ and $x_{j} \in \mathbb{Z}$ for $j \in\{1, \cdots, i\} \backslash F$, let

$$
U_{F}^{\left\{x_{j}\right\}_{j \in\{1, \cdots, i\} \backslash F}}=\prod_{j \notin F}\left\{2^{j} x_{j}\right\} \times \prod_{j \in F}\left[2^{j} x_{j}+r, 2^{j}\left(x_{j}+1\right)-r\right] \text { and }
$$

$$
\mathscr{A}_{i}=\left\{U_{F}^{\left\{x_{j}\right\}_{j \in\{1, \cdots, i\} \backslash F}} \mid F \subset\{1, \cdots, i\} \text { with }|F|=n \text { and } x_{j} \in \mathbb{Z} \text { for } j \in\{1, \cdots, i\} \backslash F\right\} .
$$

Then $A_{i} \doteq \bigcup \mathscr{A}_{i}=\left\{\left(x_{1}, \ldots, x_{i}\right) \in X_{\omega+n}^{(i)}||\left\{j \mid d\left(x_{j}, 2^{i} \mathbb{Z}\right) \geq r\right\} \mid \leq n\right\}=X_{\omega+n} \backslash N_{r}\left(X_{\omega+n-1}\right)$. Using the definition, $d\left(A_{p}, A_{q}\right)>r$ for $p \neq q \in \mathbb{N}$ and $p, q \geq i_{0}$, so $\bigcup_{i=i_{0}}^{\infty} \mathscr{A}_{i}$ is still an $r$-disjoint family of subsets such that asdim $(V) \leq n$ uniformly for every $V \in \bigcup_{i=i_{0}}^{\infty} \mathscr{A}_{i}$. So $\operatorname{decodim}\left(X_{\omega+n}\right) \leq \omega+n$. 
Example 3.21. $\operatorname{decodim}\left(Y_{2 \omega}\right) \leq 2 \omega$.

Proof. For $R>0$, since $Y_{2 \omega}=a s \bigsqcup_{k=1}^{\infty} Y_{\omega+k}$, then $\mathscr{U}=\left\{\{x\} \mid x \in a s \bigsqcup_{k=R+1}^{\infty} Y_{\omega+k}\right\}$ is a $R$-disjoint uniformly bounded subsets family, and $\bigcup \mathscr{U}=a s \bigsqcup_{k=R+1}^{\infty} Y_{\omega+k}$. By definition $Y_{\omega+k} \subset X_{\omega+R}$ for $k \leq R$, so there exists $m \in \mathbb{N}$ such that $\operatorname{decodim}\left(a s \bigsqcup_{k=1}^{R} Y_{\omega+k}\right) \leq \omega+m$, which implies $\operatorname{decodim}\left(Y_{2 \omega}\right) \leq 2 \omega$.

Definition 3.22. ([11]) A metric family $\mathscr{X}$ is $r$-decomposable over a metric family $\mathscr{Y}$ if every $X \in \mathscr{X}$ admits a decomposition

$$
X=X_{0} \cup X_{1}, X_{i}=\bigsqcup_{r \text {-disjoint }} X_{i j},
$$

where each $X_{i j} \in \mathscr{Y}$. It is denoted by $\mathscr{X} \stackrel{r}{\rightarrow} \mathscr{Y}$.

Definition 3.23. ([11])

(1) Let $\mathscr{D}_{0}$ be the collection of bounded families: $\mathscr{D}_{0}=\{\mathscr{X}: \mathscr{X}$ is uniformly bounded $\}$.

(2) Let $\alpha$ be an ordinal greater than 0 , and let $\mathscr{D}_{\alpha}$ be the collection of metric families decomposable over $\bigcup_{\beta<\alpha} \mathscr{D}_{\beta}$ :

$$
\mathscr{D}_{\alpha}=\left\{\mathscr{X}: \forall r>0, \exists \beta<\alpha, \exists \mathscr{Y} \in \mathscr{D}_{\beta} \text {, such that } \mathscr{X} \stackrel{r}{\rightarrow} \mathscr{Y}\right\} .
$$

Definition 3.24. ([11]) A metric family $\mathscr{X}$ has finite decomposition complexity if there exists a countable ordinal $\alpha$ such that $\mathscr{X} \in \mathscr{D}_{\alpha}$.

Remark 3.25. We view a single metric space $X$ as a metric family with a single element $\{X\}$.

Lemma 3.26. ([11]) A metric space $X$ has finite asymptotic dimension if and only if $\{X\} \in \mathscr{D}_{n}$ for some $n \in \mathbb{N}$.

By imitating the proofs of Proposition 3.8 in [3], we easily obtain the following lemma.

Lemma 3.27. For a metric famliy $\mathscr{X}=\left\{X_{\alpha}\right\}_{\alpha \in \mathfrak{A}}$, asdim $\left(X_{\alpha}\right) \leq n$ uniformly implies $\left\{X_{\alpha}\right\}_{\alpha \in \mathfrak{A}} \in$ $\mathscr{D}_{n}$.

Proposition 3.28. For a metric space $X$ and an ordinal number $\xi$, decodim $(X) \leq \xi$ implies $\{X\} \in \mathscr{D}_{\xi}$.

Proof. We will prove it by an induction on $\xi$.

- For $\xi \in \mathbb{N}$, decodim $(X) \leq \xi$ implies $X$ has finite asymptotic dimension. Then $\{X\} \in \mathscr{D} \xi$ by Lemma 3.26.

- For $\xi=\omega$, for every $r>0$, since $\operatorname{decodim}(X) \leq \omega$, there is an $r$-disjoint and uniformly bounded family $\mathscr{U}$ of subsets of $X$ such that $\operatorname{asdim}(X \backslash \cup \mathscr{U}) \leq n$ for some $n \in \mathbb{N}$. By Lemma 3.26, $\{X \backslash \bigcup \mathscr{U}\} \in \mathscr{D}_{n}$. Let $\mathscr{Y}=\{U \mid U \in \mathscr{U}\} \bigcup\{X \backslash \bigcup \mathscr{U}\}$. Then $\mathscr{Y} \in \mathscr{D}_{n}$ and $\{X\} \stackrel{r}{\rightarrow} \mathscr{Y}$, which implies that $\{X\} \in \mathscr{D} \omega$.

- For $\xi>\omega$, assume that $\operatorname{decodim}(Y) \leq \gamma$ implies $\{Y\} \in \mathscr{D}_{\gamma}$ for every metric space $Y$ and for every $\gamma<\xi$. Now let $X$ be a metric space with $\operatorname{decodim}(X) \leq \xi$. For every $r>0$, there is an $r$-disjoint family $\mathscr{U}$ of subsets of $X$ with $\operatorname{asdim}(U) \leq n(\xi)$ uniformly for every $U \in \mathscr{U}$, such that $\operatorname{decodim}(X \backslash \cup \mathscr{U}) \leq \beta$ for some ordinal number $\beta<\xi$. By assumption, $\{X \backslash \cup \mathscr{U}\} \in \mathscr{D}_{\beta}$. By Lemma 3.27, $\mathscr{U} \in \mathscr{D}_{n(\xi)}$. Let $\mathscr{Y}=\{U \mid U \in$ $\mathscr{U}\} \bigcup\{X \backslash \bigcup \mathscr{U}\}$, then $\mathscr{Y} \in \mathscr{D}_{\beta}$ and $\mathscr{X} \stackrel{r}{\rightarrow} \mathscr{Y}$. So $\{X\} \in \mathscr{D} \xi$. 
Corollary 3.29. For any $k \in \mathbb{N}, X_{\omega+k} \in \mathscr{D}_{\omega+k}$, and $Y_{2 \omega} \in \mathscr{D}_{2 \omega}$.

\section{Funding}

This work was supported by the National Natural Science Foundation of China (Grant No.12071183).

\section{Acknowledgements}

The authors wish to thank the referees for careful reading and valuable comments.

\section{REFERENCES}

[1] M. Gromov, Asymptotic invariants of infinite groups, in: Geometric Group Theory, Vol.2, Sussex, 1991, in: Lond. Math. Soc. Lect. Note Ser., vol.182, pp. 1-295, Cambridge Univ. Press, Cambridge, 1993.

[2] G. Yu, The Novikov conjecture for groups with finite asymptotic dimension, Ann. Math. 147 (1998), $325-355$.

[3] A. Dranishnikov, M. Zarichnyi, Universal spaces for asymptotic dimension, Topol. Appl. 140 (2004), 203225.

[4] G. Bell, A. Dranishnikov, Asymptotic dimension in Bȩdlewo, Topol. Proc. 38 (2011), 209-236.

[5] A. Dranishnikov, M. Zarichnyi, Asymptotic dimension, decomposition complexity, and Havar's property C, Topol. Appl. 169 (2014), 99-107.

[6] A. Dranishnikov, Asymptotic topology, Russ. Math. Survey 55 (2000), 1085-1129.

[7] T. Radul, On transfinite extension of asymptotic dimension, Topol. Appl. 157 (2010), 2292-2296.

[8] J. Zhu, Y. Wu, A metric space with its transfinite asymptotic dimension $\omega+1$, Topol. Appl. 273 (2020), 107115.

[9] Y. Wu, J. Zhu, Classification of metric spaces with infinite asymptotic dimension, Topol. Appl. 238 (2018), 90-101.

[10] E. Guentner, R. Tessera, G. Yu, A notion of geometric complexity and its application to topological rigidity, Invent. Math. 189 (2012), 315-357.

[11] E. Guentner, R. Tessera, G. Yu, Discrete groups with finite decomposition complexity, Groups Geom. Dyn. 7 (2013), 377-402.

[12] M. Satkiewicz, Transfinite Asymptotic Dimension, arXiv:1310.1258v1, 2013.

[13] R. Engelking, Theory of Dimensions: Finite and Infinite, Heldermann Verlag, 1995.

[14] Y. Wu, J. Zhu, A metric space with transfinite asymptotic dimension $2 \omega+1$, Chin. Ann. Math. (2021), in press.

[15] Y. Wu, J. Zhu, The relationship between asymptotic decomposition properties, Topol. Appl. 292 (2021), 107623 . 